\title{
Medical Prophylaxis of Post-Surgical Crohn's Disease Recurrence: Towards Timely Anti-TNF Therapy
}

\author{
Adam C. Fields $^{1} \cdot$ Nelya Melnitchouk $^{1}$
}

Published online: 10 August 2018

○) Springer Science+Business Media, LLC, part of Springer Nature 2018

Crohn's disease typically affects the terminal ileum and proximal colon; approximately $50 \%$ of patients will require bowel resection due to penetrating disease or stricture within 10 years of disease diagnosis [1]. Intestinal resection for these patients is not curative with up to $90 \%$ of patients demonstrating endoscopic evidence of recurrence 1 year postoperatively [1]. Risk factors for Crohn's disease recurrence after surgical therapy include cigarette smoking, younger age, penetrating disease, shorter duration of disease prior to resection, prior surgical resections, and ileocolic disease [2]. Antibiotics, thiopurines, and steroids all have variable efficacy in reducing postoperative recurrence. In 2009, Regueiro et al. [3] provided the first evidence that infliximab administered as a postoperative prophylactic therapy could reduce endoscopic, clinical, and histologic Crohn's disease recurrence published as a proof-of-concept randomized trial. Although only 24 patients were included in this study, 9.1\% of patients receiving infliximab within 4 weeks of surgery compared to $84.6 \%$ of patients receiving placebo had endoscopic recurrence at 1 year postoperatively. Subsequent trials have shown that the administration of anti-tumor necrosis factor (anti-TNF) agents several weeks postoperatively effectively reduces Crohn's recurrence and anti-TNF agents are often superior to thiopurines [4, 5]. In 2017, the American Gastroenterological Association (AGA) published its guidelines on the management of Crohn's disease after surgery [2] recommending anti-TNF therapy and/or thiopurines within 8 weeks of surgery as first-line pharmacological prophylaxis for disease recurrence. To date, there have been no studies assessing the percentage of high-risk Crohn's patients

Nelya Melnitchouk

nmelnitchouk@bwh.harvard.edu

Adam C. Fields

acfields@partners.org

1 Division of Colorectal Surgery, Department of Surgery, Harvard Medical School, Brigham and Women's Hospital, 75 Francis Street, Boston, MA 02115, USA actually receiving timely postoperative anti-TNF therapy and evaluating and analyzing the factors associated with delayed administration.

In this issue of Digestive Diseases and Sciences, CohenMekelburg et al. [6] set out to determine the percentage of high-risk Crohn's disease patients receiving postoperative prophylactic anti-TNF agents as well as the risk factors associated with delays in initiation of such medications. The authors hypothesized that specific patient factors, prior anti-TNF therapy, and the type of treatment center would impact the timing of postoperative prophylactic biologic therapy. To validate this hypothesis, a retrospective cohort study was carried out in 84 patients who were deemed by two independent reviewers at high risk for disease recurrence and were likely to benefit from postoperative biologic therapy. The authors found that $69 \%$ of patients had greater than a 4-week delay and $56 \%$ of patients had greater than an 8 -week delay in starting postoperative biologic prophylaxis. Moreover, the authors found that patients with public insurance were more likely to have delays in initiating biologic therapy, whereas patients receiving preoperative biologic therapy or who received care at an inflammatory bowel disease (IBD) center were more likely to have timely therapy. Two specific methodological strengths of the study are the assessment of high risk of recurrence Crohn's patients by two independent reviewers and a sensitivity analysis including immunomodulators and biologics.

This is the first study to highlight the significant percentage of high-risk Crohn's disease patients that have delayed postoperative biologic therapy. It is important to note, however, that the AGA Crohn's disease postsurgical management guidelines were not published until 2017, after the current investigation's completion (2016). Therefore, although the results of the Regueiro study examining the efficacy of postoperative biologic prophylaxis were published in 2009, the impetus to start biologics postoperatively may have been provider-specific as there were no formal published guidelines available prior to 2017 . Therefore, an updated analysis 
of patients receiving postoperative biologic therapy after 2017 is an important next step in determining adherence to the AGA recommendations. Further, the optimal postoperative timing of initiating prophylactic biologic therapy has not been determined. Regueiro et al. (2009) used a 4-week cutoff, while the current study assessed both 4 - and 8-week time periods. The current guidelines recommend initiation of biologic therapy in high-risk patients by 8 weeks postoperatively. Further studies are needed to assess the ideal timing of prophylactic biologic initiation.

The authors found that public insurance and lack of preoperative biologic therapy were risk factors for delayed initiation of biologic therapy, whereas care at an IBD center was associated with timely initiation of biologic treatment. Medication authorization and patient resources may have contributed to the delay in starting biologics for patients receiving public insurance. Prior studies have shown that public insurance is associated with less timely access to biologic therapy in IBD patients [7]. Since a lack of preoperative biologic therapy associated with delayed postoperative therapy could have been due to intolerance of the medication prior to surgery or because this is a marker for lack of preoperative care by an IBD specialist, this risk factor for the delay in starting postoperative biologic therapy must be further studied. Large variations in IBD medical management, especially for Crohn's disease, exist, and the type of treating gastroenterologist influences treatment strategy [8].

In the current study, approximately $25 \%$ of patients were lost to follow-up at 4 weeks postoperatively; the authors state that attempts to reach this patient population were unsuccessful. Part of this loss to follow-up may be explained by the fact that only $62 \%$ of patients resided within the vicinity of the study institution; it is unclear if these patients were seen at outside institutions or with their primary gastroenterologist or if they were truly lost to follow-up. This may be another patient population at high risk for delayed initiation of biologic therapy. Close communication between the surgeon, gastroenterologist, and patient as well as preoperative plans for initiating postoperative biologic therapy may prevent this loss to follow-up.

Postoperative surgical complications must also be considered a factor in starting postoperative biologic therapy. Questions that arise are twofold: (1) do postoperative complications delay the initiation of biologic therapy and (2) is postoperative biologic therapy associated with adverse events? This study reports only one patient having a postoperative infection delaying initiation of biologic therapy; the study is not aimed at assessing complications related to biologic use and is not powered for this outcome. A recent meta-analysis by Zhao et al. (2017) illuminates this topic; initiating postoperative biologic therapy was not associated with an increased rate of adverse events [9]. Further, waiting 2-4 weeks to start biologic therapy likely provides adequate time for postoperative infectious complications to manifest.

In conclusion, the present study emphasizes the high rate of delays in starting postoperative prophylactic biologic therapy and the importance of multidisciplinary care in the management of Crohn's disease patients. Surgeons and IBD gastroenterologists must work together closely to develop postoperative treatment plans to prevent Crohn's disease recurrence. Prospective studies assessing optimal timing of biologic initiation postoperatively as well as the efficacy of newer biologic agents are warranted. Particular attention to patient populations at high risk of delay in starting biologics is needed to reduce postsurgical disease recurrence.

\section{Compliance with ethical standards}

Conflict of interest None.

\section{References}

1. Regueiro M, Velayos F, Greer JB, et al. American Gastroenterological Association Institute technical review on the management of Crohn's disease after surgical resection. Gastroenterology. 2017;152:277-295.

2. Nguyen GC, Loftus EV, Hirano I, et al. American Gastroenterological Association Institute guideline on the management of Crohn's disease after surgical resection. Gastroenterology. 2017; 152:271-275.

3. Regueiro M, Schraut W, Baidoo L, et al. Infliximab pre- vents Crohn's disease recurrence after ileal resection. Gastroenterology. 2009;136:441.e1-450.e1 (quiz 716).

4. Armuzzi A, Felice C, Papa A, et al. Prevention of post- operative recurrence with azathioprine or infliximab in patients with Crohn's disease: an open-label pilot study. J Crohns Colitis. 2013;7:e623-e629.

5. Savarino E, Bodini G, Dulbecco P, et al. Adalimumab is more effective than azathioprine and mesalamine at preventing postoperative recurrence of Crohn's disease: a randomized controlled trial. Am J Gastroenterol. 2013;108:1731-1742.

6. Cohen-Mekelburg S, Gold S, Schneider Y, et al. Delays in initiating post-operative prophylactic biologic therapy are common among Crohn's disease patients. Dig Dis Sci. (Epub ahead of print). https://doi.org/10.1007/s10620-018-5159-4.

7. Rumman A, Candia R, Sam J, et al. Public versus private drug insurance and outcomes of patients requiring biologic therapies for inflammatory bowel disease. Can J Gastroenterol Hepatol. 2017. https://doi.org/10.1155/2017/7365937.

8. Weaver K, Sandler R, Martin C, Chen W, Anton K, Long M. Gastroenterologist practice setting in disease outcomes and quality process measures. Inflamm Bowel Dis. 2016;22:2672-2677.

9. Zhao Y, Ma T, Yan-Fang C, et al. Biologics for the prevention of postoperative Crohn's disease recurrence: a systematic review and meta-analysis. Clin Res Hepatol Gastroenterol. 2015;39:637-649. 